\title{
Manejo de la fístula anorrectal compleja. LIFT, colgajo de avance y otras técnicas
}

\author{
Alejandro Readi V. ${ }^{1}$ y Roberto Salas O. ${ }^{1}$
}

Departamento de Cirugía Hospital del Salvador. Santiago, Chile.

Recibido el 23 de abril de 2019 y aceptado para publicación el 27 de septiembre de 2019

Correspondencia a: Dr. Roberto Salas O. rgsalas@gmail.com

\section{Management of complex anorectal fistula. LIFT, endorectal advancement flap and others techniques}

Complex anal fistula are a challenge for colorectal surgeons. It is a common pathology in population. Pathogenesis is still unclear, it would be involved citokines and the process of epitelial to eesenchymal transition. The gold standard for study is MRI, its use reduces recurrences. The goal of treatment is heal the fistula without damaging the function of the sphincter and avoid recurrences. There are multiple techniques, the most accepted is ligation of intersphincteric fistula tract with cure rate over $70 \%$, with minimal impact in continence. This review includes other techniques like rectal advancement flap, fibrin glue, permacol, stem cells, anal fistula plug, video asisted anal fistula treatment, over the scope clip and fistula laser closure.

Key words: anal sepsis; complex anal fistula; aetiology; surgical treatment.

\section{Resumen}

Las fístulas anorrectales complejas son un desafío para el coloproctólogo. Son una patología frecuente que afecta la calidad de vida de los pacientes. La patogénesis aún no está clara, estarían involucradas citoquinas y el proceso de transición de epitelio a mesénquima. El gold standard para su estudio es la resonancia nuclear magnética, su uso por sí mismo disminuye la recurrencia. El objetivo del tratamiento es lograr la curación sin afectar la función del esfínter evitando las recidivas. Existen múltiples técnicas, siendo la de mayor aceptación la ligadura interesfinteriana del trayecto fistuloso, con tasa de curación sobre el 70\%, con mínimo impacto en continencia. Esta revisión incluye otras técnicas como el colgajo endorrectal de avance, uso de sellante, permacol, células madres, Anal fistula plug, Video asisted anal fistula treatment, Over the scope clip y fistula laser closure.

Palabras clave: absceso anorrectal; fístula anorrectal compleja; etiología; tratamiento quirúrgico.

\section{Introducción}

Las fístulas anorrectales (FAR) son frecuentes en la población con una prevalencia entre 8,6 a 10/100.000 al año en Europa y una relación hombre/mujer 1,8 es a $1^{1}$, Afecta la calidad de vida con ensuciamiento, ano húmedo, dolor perianal cíclico y drenaje de material purulento ${ }^{2}$. Constituyen un desafío para los coloproctólogos. Aproximadamente el 95\% son de origen criptoglandular. El resto incluye enfermedad de Crohn (EC), trauma, cirugía previa y patología tumoral ${ }^{3}$. Esta revisión busca presentar las técnicas para el tratamiento y sus resultados. Para esto se realizó búsqueda en PUBMED de los términos "anal fistula", "surgical treatment", "Pathogenesis, "Fistula-in-ano" entre enero de 2012 y julio de 2019 en inglés y español, se incluyeron revisiones sistemáticas, metaanálisis, revisiones de sociedades científicas (position staments), presentaciones de técnicas y trabajos prospectivos. No se incluyeron trabajos retrospectivos ni reportes de caso.

\section{Factores de riesgo y etiología}

Las FAR son un trayecto fibroso recubierto por tejido granulatorio entre un orificio primario interno y uno o múltiples secundarios externos en la piel de la región perianal. 
Los factores de riesgo para desarrollar una FAR incluyen ser hombre, obesidad y tabaquismo ${ }^{3}$. Diversos factores confluyen para el desarrollo de una FAR. En los abscesos anorrectales es más frecuente la presencia de microorganismos provenientes del intestino que provenientes de la piel. El mecanismo exacto para el quiebre de las barreras fisiológicas e inmunológicas del epitelio no ha sido determinado, pero hay pistas de que tanto la Transición de Epitelio a Mesénquima (EMT), como las citoquinas pueden jugar un $\mathrm{rol}^{4}$ (Figura 1). La mayoría de esta información proviene de los estudios preclínicos en EC. La EMT se ha descrito en la cicatrización de heridas y permite pensar que las células epiteliales del intestino penetran las capas profundas de la mucosa y recto, causando daño al tejido local, formación de tubo y conexión a otros órganos. La EMT se desencadenaría por remanentes bacterianos que causarían liberación de metaloproteinasas como en EC. Por último, citoquinas proinflamatorias como TNF- $\alpha$ e IL-13 exacerban el proceso aumentando la expresión de moléculas asociadas a la invasión celular.

Con respecto a la persistencia de la FAR se ha evaluado diferentes factores. La edad, género, hormonas, stress, diabetes, medicamentos (como corticoides, AINES, quimioterapia), obesidad, consumo de alcohol, tabaquismo e innmunodeficencia se han observado en el retraso de cicatrización de heridas. Sin embargo, solo el tabaquismo y la obesidad han podido ser vinculados como factor de riesgo de recurrencia de FAR. Se evaluó la histología de las FAR, no observando relación en el grado de epite- lización con el riesgo de que la FAR persista. Se ha cultivado tejidos de las fístulas crónicas, observando la presencia de un bajo número de bacterias $(E$. coli $22 \%$, B. Fragilis $20 \%$, enterococo $16 \%$ ), por lo que concluyen que la inflamación crónica no requiere un número excesivo de estas ni organismos inusuales. Se realizó hibridación in situ, gram y microscopia electrónica a muestras tomadas de orificio fistuloso interno (OFI). Solo se encontró contenido bacteriano en 1 de 32 de fístulas. Los autores sugieren buscar explicaciones alternativas a la persistencia de las fístulas. Si bien, las bacterias podrían estar en gran parte ausente en las fístulas crónicas, sus componentes podrían continuar jugando un rol en la inflamación. Un estudio reportó la presencia de peptidoglicano (componente de la pared celular) en 9 de 10 fístulas estudiadas. Se ha observado la expresión de IL-1b (93\%), IL-8 (70\%), IL-12p40 (33\%), and TNF-a $(30 \%)$. Sin embargo, no es posible establecer una correlación entre la expresión de citoquinas y los outcomes clínicos ${ }^{3}$.

\section{Clasificación}

En las FAR clásicamente se ha utilizado la clasificación de Parks del Hospital St Mark (1979), que define cuatro tipos: fístulas interesfintéricas, transesfintéricas, supraesfintéricas y extraesfintéricas.

Las fístulas superficiales fueron agregadas posteriormente y corresponden a las fístulas que no comprometen el esfínter.

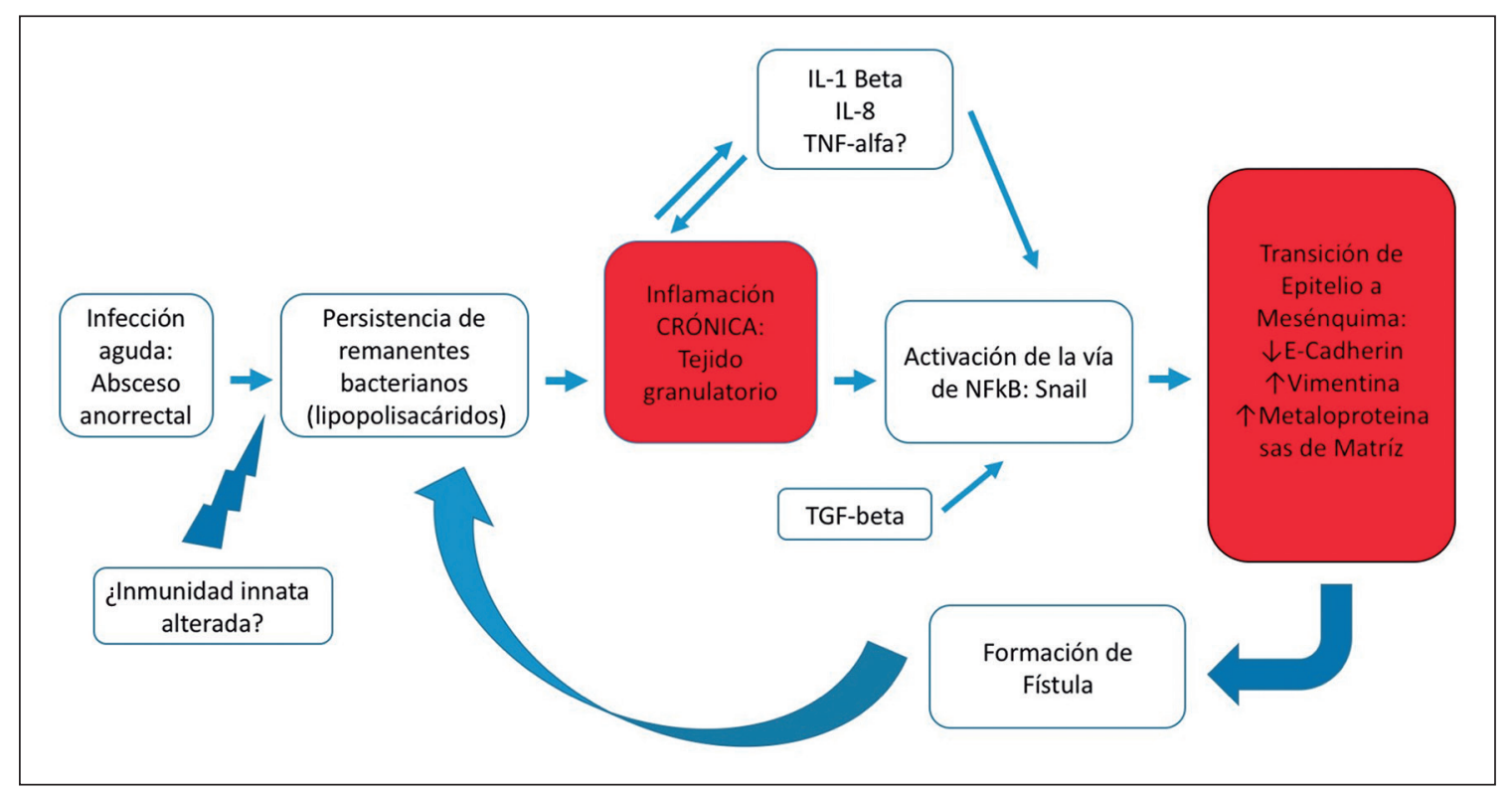

Figura 1. Modelo propuesto por Ratto et al. ${ }^{4}$ para la patogénesis de las fístulas anorrectales. 
Las fístulas complejas no fueron incluidas en la clasificación de Parks ${ }^{5}$.

Las fístulas anorrectales complejas incluyen: transesfintéricas altas, supraesfintéricas, extraesfintéricas, recurrentes, en herradura, con tractos múltiples, ubicadas en cara anterior en pacientes mujeres y las que se presenta en paciente con enfermedad inflamatoria intestinal, radiación, incontinencia preexistente o diarrea crónica ${ }^{6}$.

\section{Estudio}

La resonancia nuclear magnética (RNM) es el gold standard 5 . Está indicada en toda fístula que se sospeche compleja. Presenta concordancia sobre el $90 \%$ con los hallazgos intraoperatorios. Buchann ${ }^{7}$, observó que la recurrencia de pacientes operados con RNM fue $16 \%$ vs un $57 \%$ en los que no se utilizó.

La endosonografía tiene una exactitud cercana al $80 \%$, con concordancia cercana al $100 \%$ en fístulas simples y de $68 \%$ en fístulas altas en comparación con hallazgos quirúrgicos.

Requiere la instilación de peróxido de hidrógeno por el orificio fistuloso externo (OFE) para observar contraste del trayecto (las burbujas son ecorefringente) e incrementar la detección del $\mathrm{OFI}^{8}$. Murad-Regadas publicó su experiencia el 2018 con 73 pacientes donde describe utilidad en identificar el esfínter y la longitud de esfínter comprometido y en postoperatorio permitiría identificar falla en el tratamiento y recurrencia 9 .

\section{Tratamiento}

Las fístulas anorrectales complejas son un desafío para el coloproctólogo. El objetivo es lograr la curación del trayecto fistuloso sin alterar la función esfinteriana y evitar la posible recidiva. Se han desarrollado múltiples técnicas, algunas de las cuales se han realizado en forma combinada. Aún no existe un gold standard. A continuación revisaremos las diferentes opciones disponibles.

\section{Ligadura interesfinteriana del trayecto fistuloso (LIFT)}

LIFT fue publicada en 2007 por Dr. Rojanasakul. Consiste en la disección de espacio interesfinteriano, identificación del trayecto fistuloso, ligadura del trayecto, remoción de la fístula y curetaje del OFE.

Yassin en su revisión sistemática ${ }^{10}$ en 2013 (498 pacientes) reporta FAR 99\% criptoglandular, 94\% transesfintéricos con una tasa de éxito de $71 \%$ y un
$6 \%$ de trastorno defecatorio menor. Vergara-Fernández $z^{11}$, en 2013 (592 pacientes) reporta cierre de la fístula en $74,6 \%$ con un promedio de 5,5 semanas, sin episodios de incontinencia. La revisión y metaanálisis de Hong ${ }^{12}$ en 2014 (1.100 pacientes) reporta el cierre de la fístula en el $76,4 \%$ de los casos, $0 \%$ incontinencia sin relación entre la instalación de setón previo al procedimiento con éxito del tratamiento.

El 2015 se publicó la revisión de Sirany ${ }^{13}$, que incluyó 26 trabajos en que se recopilan 7 variaciones a la técnica de LIFT, incluyendo LIFT asociado a colgajos, con o sin escisión, con plug intrafístulas o con malla bioprotésicas en el espacio interesfintéricos, presenta cierre de la fístula entre un $47 \%$ y $95 \%$. Sin embargo, dado lo heterogéneo de los trabajos, es difícil obtener conclusiones categóricas. Cabe destacar que en este trabajo reafirma que la LIFT presenta casi nulo impacto en la continencia, reportando incontinencia entre $0 \%$ y $33 \%$.

La publicación de Kontovounisios ${ }^{14}$ en 2016, compara las tasas de éxito y adopción de diferentes técnicas (colgajos de avance, sellante, mallas de colágeno y LIFT) en un total de 133 estudios y 5.604 pacientes. Demuestra que las tasas de éxito y adopción de las diferentes técnicas van decayendo en el tiempo, sin embargo, esta tendencia es menos evidente en LIFT por lo que se podría considerar al LIFT como una técnica en consolidación.

LIFT puede actuar como "downstaging", ya que una proporción de pacientes que recidivan pasan de una fístula transesfintérica a una interesfinteriana, permitiendo fistulotomía preservando indemne el esfínter externo que originalmente pudo estar comprometido $^{8}$.

Sugrue $^{15}$, presenta un trabajo retrospectivo en que compara sellante, plugs, LIFT, dermal flap, rectal flap. Flap y LIFT presentaron los mejores resultados. Se evidenció un incremento en la utilización de LIFT del año 2009 al 2015 con buena respuesta y baja tasa de incontinencia.

En la experiencia de nuestro centro, presentada por Moreno ${ }^{16}$ el año 2019, se trató a 22 pacientes con LIFT, 16 de ellos con setón instalado dos meses antes. Presentaron una tasa de curación de $77 \%$. El $63 \%$ de la recidiva fue interesfinteriana, las cual se trataron con fistulotomía logrando un cierre total del $90 \%$. No presentaron modificaciones en el score de Wexner. No hubo significancia estadística para evaluar utilidad del setón dado el número de pacientes.

\section{Colgajo endorrectal de avance (CERA)}

Consiste en la resección de la mucosa en relación al OFI con cobertura con colgajo del defecto y resección de la porción extraesfinteriana del trayecto 
fistuloso, preservando el esfínter anal interno. Se describen tasa de éxito entre un $70 \%$ a $90 \%$ con incontinencia leve a moderada entre un $7 \%$ a $38 \%{ }^{6}$.

Balsiscueta $^{17}$, presentó en el 2017 una revisión sistemática y metaanálisis CERA en fístulas complejas. Incluyó 26 trabajos con un total de 1.655 pacientes. Describe una recurrencia global de $21 \%$ con una tasa de incontinencia global de $13,3 \%$. Los mejores resultados en relación a recurrencia se obtiene realizando colgajo de pared completa $(7,4 \%)$ en comparación con colgajo parcial (19\%) y de mucosa $(30,1 \%)$. En contraste, es mayor la incontinencia en pacientes con colgajo de pared completa $(20,4 \%)$ en comparación con pared parcial $(10,2 \%)$ y mucosa $(9,3 \%)$. En la mayoría de los casos los síntomas fueron leve. Se comparó la resección del OFE tipo Core-out y curetaje obteniendo recurrencia similar $(19 \%$ vs $21 \%)$ e incontinencia similar (14,3\% vs $12 \%)$.

Podetta $^{18}$ en 2018, describe el uso colgajo de avance de mucosa posterior a recidiva en pacientes que ya fueron sometidos colgajo previamente. El éxito al primer intento fue un $66,1 \%$, en el grupo con segundo intento $78,1 \%$. Bessi ${ }^{19}$, en el 2019 , publica una serie con 87 pacientes ( 34 con EC) con un seguimiento de 24 meses mostrando un tasa de éxito de $65,9 \%$ global y de $66,7 \%$ para EC. Rottoli ${ }^{20}$, el mismo año propone una alternativa en el abordaje mediante TAMIS (Transanal Minimally Invasive Surgery) para fístulas altas con su experiencia inicial de 4 pacientes. Se requieren estudios adicionales para recomendar esta técnica.

\section{LIFT vs CERA}

Dado que estas son las técnicas con mejores resultados se han desarrollado diferentes trabajos para definir cuál es mejor. Tan ${ }^{21}$ (2012), realizó un trabajo con 24 pacientes sometidos a LIFT vs 31 pacientes a CERA, todos tenían previamente instalado un setón. Los resultados mostraron una tasa de éxito de $62,5 \%$ para LIFT y 93,5\% para CERA (p 0,006). Stellingwerf ${ }^{22}$, publica el 2019 una revisión sistemática y metaanálisis de CERA vs LIFT en paciente con fístulas de origen criptoglandular y Crohn. 1.295 pacientes (CERA 797, 498 LIFT) con una tasa de éxito de $72,6 \%$ CERA y $69,1 \%$ LIFT y recurrencia $25,6 \%$ CERA $21,9 \%$ LIFT, ambos resultados sin diferencias significativas. En Crohn tampoco hay diferencias significativas: CERA 61 vs 53\%. CERA tiene mayor tasa de incontinencia $7,8 \%(3,3 \%-12,4 \%)$ vs $1,6 \%(0,4 \%-2,8 \%)$, por lo que concluye que no hay diferencias significativas entre ambas técnicas, pero si podría presentar mayor incontinencia CERA.

\section{Sellante}

Consiste en instilación de una preparación en el trayecto fistuloso que provee un puente para fibroblastos y crecimiento de células estromovasculares. Diversos materiales han sido utilizados, desde la propia sangre del paciente hasta una mezcla de factores de coagulación, aprotinina y calcio como también pegamentos sintéticos (cianocrilato). Dado el mínimo riesgo de incontinencia, su uso repetido es una opción atractiva en pacientes de alto riesgo de disfunción de esfínter ${ }^{8}$. La tasa de curación en los diferentes trabajos revisados por Williams tiene un rango de $14 \%$ al 94\%, con resultados contradictorios sobre beneficios a diferentes longitudes de trayecto ${ }^{8}$. Los principales estudios clínicos randomizados muestran una tasa de curación entre $40 \%-50 \%{ }^{6}$. El sellante es una técnica con éxito modesto pero con bajo riesgo de incontinencia.

\section{Permacol}

Usando el mismo principio que el sellante, permacol es una matriz acelular derivada de porcino que contiene colágeno tipo I, tipo III y elastina. Schiano di Visconte ${ }^{23}$, en 2018, comparó colgajo de avance (31 pacientes) vs permacol (21 pacientes). Ambos grupos tuvieron setón instalado un promedio de 8 semanas antes de la cirugía. La tasa de tiempo libre de enfermedad a los dos años fue de $65 \%$ para colgajo y $52 \%$ para permacol, con incontinencia de $16 \%$ para el primero y $5 \%$ para el segundo. El mismo autor realiza un trabajo multicéntrico $^{24}$ con 46 pacientes, mostrando una tasa de curación de $50 \%$ a los dos años de seguimiento sin alteración de la continencia. Existe poca evidencia hasta el momento que permita recomendar o descartar su uso.

\section{Células madre}

Las células madre son obtenidas de tejido adiposo autólogo expandido. La técnica consiste en la localización del OFI, el curetaje del tracto fistuloso, el aseo de la cavidad, cierre del OFI, resuspensión de las células madres y la inyección de las células. Se recomienda inyectar la mitad de la solución en el tejido periOFI y la otra mitad a través del OFE en la pared de la fístula en toda su extensión ${ }^{25}$. Un trabajo del año 2012 reporta una tasa de éxito de 50\% a 1 año de seguimiento ${ }^{26}$. Un trabajo multicéntrico compara el uso de células madre con uso de células madre con sellante y sellante, pudiendo recibir los grupos con células madre nueva inyección a las 12 semanas. La tasa de curación a un año fue de $57,1 \%$, $52,4 \%$ y $37,3 \%$, respectivamente sin diferencias significativa entre los tres grupos. En un trabajo en 
pacientes con EC reportan tasas de curación de 75\% hasta 2 años de seguimiento ${ }^{27}$.

Dado la escasa evidencia no es posible recomendar este tratamiento.

\section{Anal fistula plug}

Los anal fistula plug son materiales (bioprotésicos o sintéticos) usados para ocluir el trayecto fistuloso, bloqueando el OFI previniendo la entrada de material fecal. El plug provee la matriz necesaria para generar respuesta inmune y regenerativa promoviendo la reparación y curación. Los plug se degradan durante varias semanas, tiempo en el cual el proceso de reparación es establecido ${ }^{8}$.

La técnica de instalación requiere los pasos de setón, curetaje, aseo, instalación de plug y su sutura a OFI y OFE para prevenir la extrusión. La revisión sistemática de O’Riordan ${ }^{28}$ del año 2012, incluyó 20 estudios con un total de 530 pacientes (42 con EC). La tasa de extrusión fue $8,7 \%$. La tasa de éxito fue un $54 \%$ en pacientes sin EC y $55 \%$ en paciente con EC. El $2016 \mathrm{Xu}^{29}$ publica un metaanálisis comparando plug vs CERA. Incluyó 778 pacientes (CERA 314 , PLUG 218) con tasa de éxito promedio de 53\% $\pm 0,18$ vs $44 \% \pm 21 \%$, sin diferencia significativa.

Habría beneficio en la asociación de plug con LIFT (94\% curación) vs LIFT solo $(83,9 \%)^{30}$.

\section{Video asisted anal fistula treatment (VAAFT)}

VAAFT es un procedimiento que tiene dos etapas: diagnóstica y terapéutica ${ }^{31}$. Requiere de un fistuloscopio y obturador que permite identificar el OFI, los tractos secundarios y abscesos. Se introduce la cámara por el OFE y se introduce una solución de glicina-manitol para abrir el trayecto fistuloso principal y avanzar bajo visión directa. En la etapa terapéutica se realiza el curetaje, aseo y cauterización del trayecto. Posteriormente, se realiza el cierre del OFI utilizando stapler lineal o circular, OTSC, colgajo o cianocrilato. En 2017 Garg $^{32}$ publica revisión sistemática y metaanálisis proporcional de VAAFT. Incluyó 8 trabajos (786 pacientes), con una tasa de éxito de $76,01 \%$ y tasa de complicaciones de $16,2 \%$. Ningún trabajo reportó deterioro de la continencia. Emile ${ }^{33}$ publicó en 2018 una revisión sistemática y metaanálisis con 788 pacientes en que describe una tasa de recurrencia global de $17,7 \%$, con diferentes resultados según tipo de cierre del OFI: stapler 15,3\%, sutura 17,7\% y CERA $25 \%$. VAAFT es una de las técnicas nuevas más promisorias.

\section{Over the scope clip}

El tratamiento con dispositivo OTSC consiste en la inserción de un clip de una aleación elástica (ni- tinol) que ejerce una presión constante sobre el OFI y facilita el cierre de la fístula. La técnica consiste en revisión de orificios fistulosos, curetaje, aseo, escisión de $2 \mathrm{~cm}$ de anodermo alrededor de OFI para que el clip no atrape fibras sensitivas que puedan provocar dolor. Sutura en forma de U para aproximar defecto y lograr una instalación perpendicular y completa del clip. Se prueba impermeabilidad del cierre con suero fisiológico y resección core-out del OFE. Existen pocos trabajos, describen tasas de éxito entre $18 \%$ a $90 \%{ }^{34}$. Los pacientes no presentan incontinencia. Una de las complicaciones a considerar es el dolor invalidante por el clip. Faltan estudios para poder definir la utilidad y éxito de este procedimiento.

\section{Fistula laser closure (FiLac)}

El uso de láser para tratamiento de fístula fue descrito inicialmente en 1980 por Stlutski. El año 2011 Wilhelm $^{35}$ publica la utilización de láser a través de una fibra radial en todo el trayecto de la fístula, provocando una contracción del tejido y consigo un selle progresivo. Requiere también cierre de OFI con un procedimiento como colgajo de avance. Los resultados publicados por Giamundo ${ }^{36}$ en 2013 presentan una tasa de éxito de $71,4 \%$ (25 pacientes). Ningún paciente reportó incontinencia. En 2017 Wilhelm $^{37}$ reporta su experiencia con 5 años de FiLaC: 117 pacientes, con una mediana de seguimiento de 24,5 meses, 13 pacientes con EC. Todos sin incontinencia. La tasa de curación primaria fue $64,1 \%$, siendo $63,5 \%$ en criptoglandular y $69,2 \%$ en EC. En el caso de falla se realizó un segundo procedimiento (Re-FiLaC, fistulectomía con preservación de esfínter o fistulotomía) logrando curación en $103 / 177$ pacientes $(88 \%), 85,5 \%$ en criptoglandular y $92,3 \%$ en EC.

Dado que no tiene efecto en la continencia y se asocia a buenos resultados es una opción interesante a considerar principalmente en paciente con EC.

\section{Conclusiones}

Las FAR complejas siguen siendo un desafío para el cirujano colorrectal. La etiología de la recurrencia aún no es clara. Son múltiples las técnicas descritas, sin contar aún con un Gold Standard, siendo las técnicas con mejor resultados LIFT y CERA (Tabla 1). El resto de las alternativas tienen baja tasa de éxito o escasos estudios que avalen su uso, teniendo que tener en consideración su utilidad en caso de altísimo riesgo de incontinencia. 
Tabla 1. Resumen de tasa de éxito por técnicas

\begin{tabular}{|c|c|c|}
\hline Técnica & Publicación & Tasa de éxito \\
\hline LIFT & $\begin{array}{l}\text { Yassin } 2013 \\
\text { Vergara-Fernández } 2013 \\
\text { Hong } 2013 \\
\text { Moreno } 2019 \\
\text { Tan } 2012 \\
\text { Stellingwerf } 2019\end{array}$ & $\begin{array}{l}71 \% \\
74,50 \% \\
76,40 \% \\
77 \% \\
63 \% \\
69 \%\end{array}$ \\
\hline $\mathrm{LIFT}+\mathrm{Co}$ & $\begin{array}{l}\text { gajos y/o plug } \\
\text { Sirany } 2015\end{array}$ & $47-95 \%$ \\
\hline CERA & $\begin{array}{l}\text { Amato } 2015 \\
\text { Balsiscueta } 2017 \\
\text { Podetta } 2018 \\
\text { Bessi } 2019 \\
\text { Tan } 2012 \\
\text { Stellingwerf } 2019\end{array}$ & $\begin{array}{l}70-90 \% \\
79 \% \\
66,10 \% \\
65,90 \% \\
93,50 \% \\
72,60 \%\end{array}$ \\
\hline Sellante & $\begin{array}{l}\text { Williams } 2018 \\
\text { Amato } 2015\end{array}$ & $\begin{array}{l}14-94 \% \\
40-50 \%\end{array}$ \\
\hline Permacol & Schiano 2019 & $50 \%$ \\
\hline Células m & $\begin{array}{l}\text { dre } \\
\text { Herreros } 2012 \\
\text { Cho } 2015\end{array}$ & $\begin{array}{l}50 \% \\
57,10 \%\end{array}$ \\
\hline Anal fistul & $\begin{array}{l}\text { Plug } \\
\text { O'Riordan } 2012 \\
\text { Xu } 2016\end{array}$ & $\begin{array}{l}54 \% \\
44 \%\end{array}$ \\
\hline VAAFT & Garg 2017 & $76 \%$ \\
\hline OTSC & Marinello & $18-90 \%$ \\
\hline FiLAC & $\begin{array}{l}\text { Giamundo } 2013 \\
\text { Wilhelm } 2017\end{array}$ & $\begin{array}{l}71,40 \% \\
64,10 \%\end{array}$ \\
\hline
\end{tabular}

LIFT: Ligadura del trayecto interesfintérico, CERA: Colgajo endorrectal de avance, VAAFT Video asisted anal fistula treatment, OTSC: Over the scope clip, FiLAC: Fistula Laser Closure.

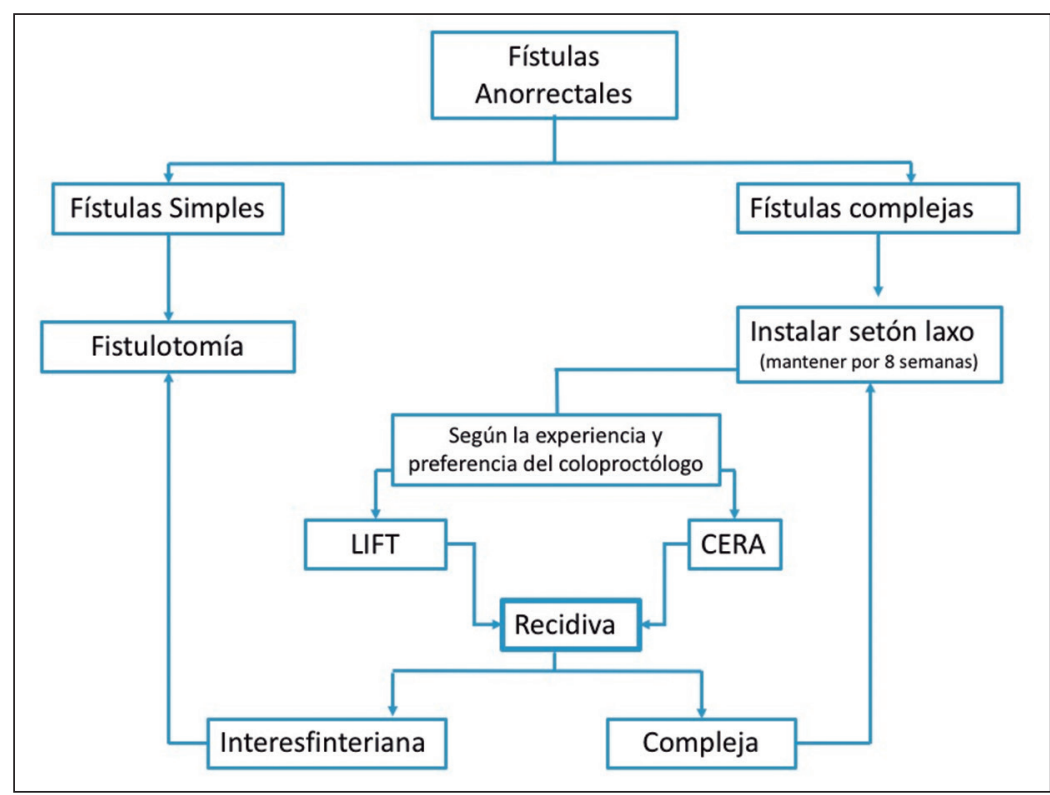

Figura 2. Esquema propuesto para manejo de fístulas. LIFT: Ligadura del trayecto interesfinteriano; CERA: Colgajo endorrectal de avance; RNM: Resonancia nuclear magnética.

Proponemos en esta revisión un esquema de toma de decisiones para el manejo de fístulas anorrectales (Figura 2).

\section{Responsabilidades éticas}

Protección de personas y animales. Los autores declaran que para esta investigación no se han realizado experimentos en seres humanos ni en animales.

Confidencialidad de los datos. Los autores declaran que en este artículo no aparecen datos de pacientes.

Conflictos de interés: no hay.

\section{Bibliografía}

1. Cadeddu F, Salis F, Lisi G, Ciangola I, Milito G. Complex anal fistula reamins a challenge for colorectal surgeon. Int J Colorectal Dis. 2015;30:595-03.

2. Owen HA, Buchanan GN, Schizas A, Cohen R, Williams AB. Quality of life with anal fistula. Am R Coll Surg Engl. 2016;98:334-38.

3. Sugrue J, Nordenstam J, Abcarian H, Bartholomew A, Schwartz J, Mellgren A, et al. Pathogenesis and persistence of cryptoglandular anal fistula: a systematic review. Tech Coloproctol.

$$
\text { 2017;21:425-32. }
$$

4. Ratto C, Litta F, Lucchetti D, Parello A, Boninsegna A, Arena V, et al. Immunopathological characterization of cryptoglandular anal fistula: a pilot study investigating its pathogenesis. Colorectal Dis. 2016;18:436-44.

5. Jhaveri K, Thipphavong S, Guo L, Harisinghani M. MR Imaging of Perianal Fistulas. Radio Clin N Am. 2018;56:77589.

6. Amato A, Bottini C, De Nardi P, Giamundo P, Lauretta A, Realis Luc A, et al. Evaluation and management of perianal abscess and anal fistula: a consensus statement developed by the Italian Society of Colorectal Surgery (SICCR). Tech Coloproctol. 2015;19:595-606.

7. Buchanan GN, Halligan S, Bartram CI, Williams AB, Tarroni D, Cohen CR. Clinical examination, endosonography, and MR imaging in preoperative assessment of fistula in ano: comparison with outcome-based reference standard. Radiology 2004;233:674-81.

8. Williams G, Williams A, Tozer P, Phillips R, Ahmad A, Jayne D, et al. The treatment of anal fistula: second ACPGBI Position Statement-2018. Colorectal Dis. 2018;20:5-31. 
9. Murad-Regadas SM, Regadas FS, Holanda EC, Veras LB, Vilarino AS, Lopes M. Can Three-dimensional anorectal ultrasonograhpy be included as a diagnostic tool for the assessment of anal fistula before and after surgical treatment? Arq. Gastroenterol. 2018; 55 (Suppl 1):1824.

10. Yassin NA, Hammond TM, Lunniss PJ, Phillips RK. Ligation of the intersphincteric fistula tract in the management of anal fistula. A systematic review. Colorectal Dis 2013;15:527-35.

11. Vergara-Fernández O, Espino-Urbina LA. Ligation of intersphincteric fistula tract: what is the evidence in a review? World $\mathrm{J}$ Gastroenterology 2013;19:6805-13.

12. Hong KD, Kang S, Kalaskar S, Wexner SD. Ligation of intersphincteric fistula tract (LIFT) to treat anal fistula: systematic review and meta-analysis. Tech Coloproctol. 2014;18:685e691.

13. Syrani AM, Nygaard R, Morken J. The Ligation of the Intersphintecteric Fistula Tract Procedure for Anal Fistula: A Mixed Bag of Results. Dis Colon Rectum 2015;58:604-12.

14. Kontovounisios C, Tekkis P, Tan E, Rasheed S, Darzi A, Wexner SD. Adoption and success rates of perineal procedures for fistula-in-ano: a systematic review. Colorectal Dis. 2016;18:441e458.

15. Sugrue J, Mantilla N, Abcarian A, Kochar K, Marecik S, Chaundhry V, et al. Sphincter-Sparing Anal Fistula Repair: Are We Getting Better? Dis Colon Rectum 2017;60:1071-7.

16. Moreno Baeza N, Readi Vallejos A, Melkonian Tumani E, Capona Pérez R, Martínez Mardones M. Ligadura interesfinteriana del trayecto fistuloso (LIFT) como alternativa de tratamiento a una fístula perianal compleja. Rev Cirugía 2019;71:42-46.

17. Balciscueta $Z$, Uribe N, Balciscueta I, Andreu-Ballester JC, García-Granero E. Rectal advancement flap for the treatment of complex cryptoglandular anal fistulas: a systematic review and meta-analysis. Int J Colorectal Dis. 2017;32:599-609.

18. Podetta, M, Scarpa CR, Zufferey G, Skala K, Ris F, Schmit L, et al. Mucosal advancement flap for recurrent complex anal fistula: a repeatable procedure. International Journal of Colorectal Disease 2018;34,197-200.
19. Bessi G, Siproudhis L, Merlini l'Héritier A, Wallenhorst T, Le Balc'h E, Bouguen $\mathrm{G}$, et al. Advancement flap procedure in Crohn and non-Crohn perineal fistulas: a simple surgical approach. Colorectal Dis. 2019;21:66-72.

20. Rottoli M, Di Simone M, Poggioli G. TAMIS-Flap Technique: Full-thickness Advancement Rectal Flap for High Perianal Fistulae Performed Through Transanal Minimally Invasive Surgery. Surgical Laparoscopy, Endoscopy \& Percutaneous Techniques 2019;29:53-6.

21. Tan K, Alsuwaigh R, Tan AM, Tan IJ, Liu X, Koh DC, et al. To LIFT or to flap? Which surgery to perform following seton insertion for high anal fistula? Diseases of the colon and rectum 2012;55;1273-7.

22. Stellingwerf ME, van Praag EM, Tozer PJ, Bemelman WA, Buskens CJ. Systematic review and meta-analysis of endorectal advancement flap and ligation of the intersphincteric fistula tract for cryptoglandular and Crohn's high perianal fistulas. BJS Open 2019;3:231-41.

23. Schiano di Visconte M, Bellio G. Comparison of porcine collagen paste injection and rectal advancement flap for the treatment of complex cryptoglandular anal fistulas: a 2-year follow-up study. Int J Colorectal Dis. 2018;33.1723-31.

24. Schiano di Visconte M, Braini A, Moras L, Brusciano L, Docimo L, Bellio G. Permacol Collagen Paste Injection for Treatment of Complex Cryptoglandular Anal Fistulas: An Observational Cohort Study With a 2-Year Follow-up. Surgical Innovation 2019;26:168-79.

25. Georgiev-Hristov T, Guadalajara H, Herreros MD, Lightner AL, Dozois EJ, García-Arranz M, et al. A Step-By-Step Surgical Protocol for the treatment of Perianal Fistula with Adipose-Derived Mesenchymal Stem Cells. J Gastrointest Surg. 2018;22:2003-12.

26. Herreros MD, García-Arranz M, Guadalajara H, de-la-Quintana P, GarcíaOlmo D. Autologous expanded adiposederived stem cells for the treatment of complex cryptoglandular perianal fistulas: a phase III randomized clinical trial (FATT 1: fistula Advanced Therapy Trial 1) and long-term evaluation. Diseases of the colon and rectum 2012;55:762-72 .

27. Cho YB, Park KJ, Yoon SN, Song KH, Kim DS, Jung SH, et al. Long-term results of adipose-derived stem cell therapy for the treatment of Crohn's fistula. Stem Cells Transl Med. 2015;4:532-7.

28. O'Riordan JM, Datta I, Jhonston C, Baxter NA. Systematic Review of the Anal Fistula Plug for Patient Whith Crohn's and Non-Crohn's Related Fistulain-ano. Dis Colon Rectum 2012;55:351-8.

29. Xu Y, Tang W. Comparison of an anal fistula plug and mucosa advancement flap for complex anal fistulas: a meta-analysis. ANZ Journal of Surgery 2016;86:978-82.

30. Han JG, Wang ZJ, Zheng Y, Chen CW, Wang XQ, Che CM, et al. Ligation of inter- sphincteric fistula tract $v s$ ligation of the intersphincteric fistula tract plus a bioprosthetic anal fistula plug procedure in patients with transsphincteric anal fistula: early results of a multicenter prospective randomized trial. Ann Surg. 2016;264:917-22.

31. Jiang HH, Liu HL, Li Z, Xiao YH, Li AJ, Chang Y, et al. Video-Assisted Anal Fistula Treatment (VAAFT) for Complex Anal Fistula: A Preliminary Evaluation in China. Med Sci Monit. 2017;23:2065-71.

32. Garg P, Singh P. Video-Assisted Anal Fistula Treatment (VAAFT) in Cryptoglandular fistula-in-ano: A systematic review and proportional metaanalysis. International Journal of Surgery 2017;46:85-91.

33. Emile SH, Elfeki H, Shalaby M, Sakr A. A Systematic review and meta-analysis of the efficacy and safety of video-assisted anal fistulatreatment (VAAFT). Surg Endosc. 2018;32:2084-93.

34. Marinello F, Kraft M, Ridaura N, Vallribera F, Espín E. Tratamiento de la fístula anal mediante clip con el dispositivo OTASC: resultados a corto plazo. Cirugía Española 2018;96:369-74.

35. Wilhelm A. A new technique for sphincter-preserving anal fistula repair using a novel radial emitting laser probe. Tech Coloproctol. 2011;15:445-9.

36. Giamundo P, Geraci M, Tibaldi L, Valente M. Closure of fistula-in-ano with laserFilaC: an effective novel sphincter-saving procedure for complex disease. Colorectal Dis. 2014;16:110-5.

37. Wilhelm A, Fiebig A, Krawczak M. Five years of experience with the $\mathrm{FiLaC}^{\mathrm{TM}}$ laser for fistula-in-ano management: longterm follow-up from a single institution. Tech Coloproctol. 2017;21:269-76. 